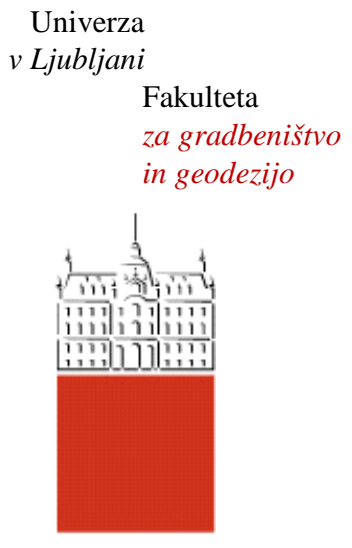

Jamova 2

1000 Ljubljana, Slovenija http://www3.fgg.uni-lj.si/

DRUGG - Digitalni repozitorij UL FGG http://drugg.fgg.uni-lj.si/

Ta članek je avtorjeva zadnja recenzirana različica, kot je bila sprejeta po opravljeni recenziji.

Prosimo, da se pri navajanju sklicujete na bibliografske podatke, kot je navedeno:

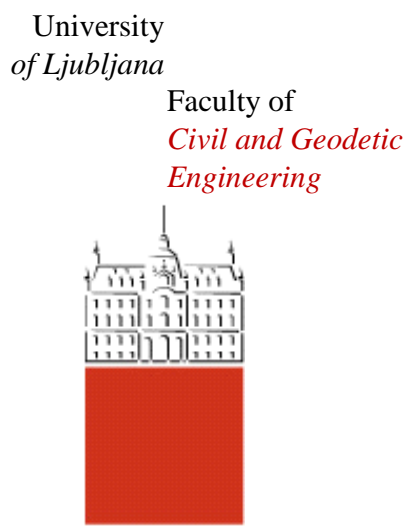

Jamova 2

SI - 1000 Ljubljana, Slovenia http://www3.fgg.uni-lj.si/en/

DRUGG - The Digital Repository http://drugg.fgg.uni-lj.si/

This version of the article is author's manuscript as accepted for publishing after the review process.

When citing, please refer to the publisher's bibliographic information as follows: 


\title{
A Comparison of the Effectiveness of Using the Meshless Method and the Finite Difference Method in Geostatistical Analysis of Transport Modelling
}

\author{
Leopold Vrankar \\ Slovenian Nuclear Safety Administration \\ Železna cesta 16, 1001 Ljubljana, Slovenia \\ leopold.vrankar@gov.si \\ Goran Turk \\ University of Ljubljana, Faculty of Civil and Geodetic Engineering \\ Jamova cesta 2, 1000 Ljubljana, Slovenia \\ gturk@fgg.uni-lj.si \\ Franc Runovc \\ University of Ljubljana, Faculty of Natural Sciences and Engineering \\ Aškerčeva cesta 12, 1000 Ljubljana, Slovenia \\ franc.runovc@uni-lj.si
}

\begin{abstract}
The disposal of radioactive waste in geological formations is of great importance for nuclear safety. The general reliability and accuracy of transport modelling depends predominantly on input data such as hydraulic conductivity, water velocity, radioactive inventory, and hydrodynamic dispersion. The most important input data are obtained from field measurements, but they are not available for the entire region of interest. One way to study the spatial variability of hydraulic conductivity is geostatistics. The numerical solution of partial differential equations (PDEs) has usually been obtained by finite difference methods (FDM), finite element methods (FEM), or finite volume methods (FVM). These methods require a mesh to support the localized approximations. The multiquadric (MQ) radial basis function method is a recent meshless collocation method with global basis functions. Solving PDEs using radial basis function (RBF) collocations is an attractive alternative to these traditional methods because no tedious mesh generation is required. We compare the meshless method, which uses radial basis functions, with the traditional finite difference scheme. In our case we determine the average and standard deviation of
\end{abstract}


radionuclide concentration with regard to spatial variability of hydraulic conductivity that was modelled by a geostatistical approach.

Key words: Transport modelling, Kansa method, Radial basis function, Geostatistics

\section{INTRODUCTION}

The objective of geological disposal of radioactive waste is to remove it from the environment and to ensure that any release remains within accepted limits. The modelling of radionuclide transport through the geosphere is necessary in the safety assessment of repositories for radioactive waste. The numerical solution of partial differential equations has been usually obtained by different types of numerical methods which usually need a support mesh.

Recent research on the numerical method has focused on the idea of using a meshless or mesh-free (gridless) methodology for the numerical solution of PDEs. One of the common characteristics of all mesh-free methods is their ability to construct functional approximation or interpolation entirely from information at a set of scattered nodes, among which there is no relationship. The best-known are the smoothed particle hydrodynamics method [1], the diffuse element method [2], the element-free Galerkin method [3], the reproducing kernel particle method [4], the partition of unity method [5], the hp-clouds method [6], the finite point method [7] and the meshless local Petrov-Galerkin method [8].

During the past decade, increasing attention has been given to the development of meshless methods using RBFs for the numerical solution of PDEs. There are two major developments in this direction. The first is the method of fundamental solutions (MFS) coupled with the dual reciprocity method (DRM), which evolved from the dual reciprocity boundary element method (DRBEM) [9]. More details about MFS can be found in review papers [10] and [11]. RBFs played a key role in the theoretical establishment and applications in the development of the DRM. With the combined features of the MFS and DRM, a meshless numerical scheme for solving PDEs has been achieved.

The second meshless method using RBFs is the Kansa method [12], [13], where the RBFs are directly implemented for the approximation of the solution of PDEs introducing the concept of solving PDEs, using radial basic functions for hyperbolic, parabolic and elliptic PDEs. A key feature of the RBF method is that it does not need a grid. Hon et al. further extended the Kansa method to the numerical solutions of various ordinary problems, as well as PDEs, for example, the biphasic mixture model for tissue engineering problems [14]. In contrast to the MFS-DRM boundary method, the Kansa method is considered to be a domain type method, which has many features similar to the finite 
element method. Since the resultant coefficient matrix of the Kansa method is not symmetric, Fasshauer [15] proposed the Hermite type method, which can generate a symmetric coefficient matrix which guarantees that the related linear equations are solvable. Strong development has taken place in this direction. However, as far as numerical performance is concerned, there is not much difference between the two approaches. Even though the Hermite approach is more theoretically robust, the Kansa method is much easier to implement.

In our case, we use the Kansa method, which uses radial basis functions and the traditional finite difference scheme. In this study we determine the average and standard deviations of radionuclide concentrations with regard to variable hydraulic conductivity modelled by a geostatistical approach. Using this approach, the general applicability, reliability and sensitivity of the Kansa meshless method and the finite difference method in transport modelling are verified.

\section{GEOSTATISTICS}

Many processes are inherently uncertain, and this uncertainty is handled through the use of stochastic realizations. The goal of stochastic simulation is to reproduce geological texture in a set of equiprobable simulated realizations. In mathematical terms, the most convenient method for simulation is sequential Gaussian simulation, because all successive conditional distributions from which simulated values are drawn are Gaussian with parameters determined by the solution of a simple kriging system.

Kriging (named after D. G. Krige, a South African mining engineer and pioneer in the application of statistical techniques to mine evaluation) is a collection of generalized linear regression techniques for minimizing an estimation variance defined from a prior model for a covariance (semivariogram).

\section{RADIAL BASIS FUNCTIONS}

A radial basis function is a function $\phi_{j}(\mathbf{x})=\phi\left(\left\|\mathbf{x}-\mathbf{x}_{j}\right\|\right)$, which depends only on the distance between $\mathbf{x} \in \mathbf{R}^{\mathbf{d}}$ and a fixed point $\mathbf{x}_{j} \in \mathbf{R}^{\mathbf{d}}$. Here, $\phi$ is continuous and bounded on any bounded sub-domain $\Omega \subseteq \mathbf{R}^{\mathbf{d}}$. Let $r$ denote the Euclidean distance between any pair of points in the domain $\Omega$. 
The commonly used radial basis functions are:

$$
\begin{aligned}
\phi(r) & =r, & & \text { linear, } \\
\phi(r) & =r^{3}, & & \text { cubic, } \\
\phi(r) & =r^{2} \log r, & & \text { thin-plate spline, } \\
\phi(r) & =e^{-\alpha r^{2}}, & & \text { Gaussian, } \\
\phi(r) & =\left(r^{2}+c^{2}\right)^{\frac{1}{2}}, & & \text { multiquadric, } \\
\phi(r) & =\left(r^{2}+c^{2}\right)^{-\frac{1}{2}}, & & \text { inverse multiquadric. }
\end{aligned}
$$

In our case we used multiquadric (MQ) and inverse multiquadric radial basis functions. The MQ method was first introduced by Hardy [16]. The parameter $c>0$ is a shape parameter controlling the fitting of a smoothing surface to the data.

Since Kansa successfully modified the radial basis functions for solving PDEs of elliptic, parabolic, and hyperbolic types, more and more computational tests have shown that this method is feasible for solving various PDEs.

To introduce RBF collocation methods, we consider a PDE in the form of

$$
\begin{gathered}
L u(\mathbf{x})=f(\mathbf{x}) \quad \text { in } \quad \Omega \subset \mathbf{R}^{\mathbf{d}}, \\
B u(\mathbf{x})=g(\mathbf{x}) \quad \text { on } \quad \partial \Omega,
\end{gathered}
$$

where $d$ is the dimension, $\partial \Omega$ denotes the boundary of the domain $\Omega, L$ is the differential operator on the interior, and $B$ is an operator that specifies the boundary conditions of the Dirichlet, Neumann or mixed type. Both $f$ and $g$ are given functions mapping $\mathbf{R}^{\mathbf{d}} \rightarrow \mathbf{R}$.

Using Kansa's asymmetric multiquadric collocation method, the unknown PDE solution $u$ is approximated by RBFs in the form

$$
u(\mathbf{x}) \approx U(\mathbf{x})=\sum_{j=1}^{N} \alpha_{j} \phi_{j}(\mathbf{x})+\sum_{l=1}^{M} \gamma_{l} v_{l}(\mathbf{x}),
$$

where $\phi_{j}(\mathbf{x})=\phi\left(\left\|\mathbf{x}-\mathbf{x}_{j}\right\|\right)$, and $\phi$ can be any radial basis function from the list, $v_{1}, \ldots, v_{M} \in \Pi_{m}^{d}$ is a polynomial of degree $m$ or less, $M:=\left(\begin{array}{c}m-1+d \\ d\end{array}\right)[17]$ and $\|\cdot\|$ indicates the Euclidean norm. Let $\left\{\left(\mathbf{x}_{j}\right)\right\}_{j=1}^{N}$ be the $N=N_{I}+N_{B}$ collocation points in $\Omega \cup \partial \Omega$. We assume the collocation points are arranged in 
such a way that the first $N_{I}$ points are in $\Omega$, whereas the last $N_{B}$ points are on $\partial \Omega$. To solve for the $N+M$ unknown coefficients, $N+M$ linearly independent equations are needed. Ensuring that $U$ satisfies (1) and (2) at the collocation points results in a good approximation of the solution $u$. The first $N$ equations are given by

$$
\begin{aligned}
& \sum_{j=1}^{N} \alpha_{j} L \phi_{j}\left(\mathbf{x}_{i}\right)=f\left(\mathbf{x}_{i}\right) \quad \text { for } \quad i=1, \ldots, N_{I} \\
& \sum_{j=1}^{N} \alpha_{j} B \phi_{j}\left(\mathbf{x}_{i}\right)=g\left(\mathbf{x}_{i}\right) \quad \text { for } \quad i=N_{I}+1, \ldots, N_{I}+N_{B}
\end{aligned}
$$

The last $M$ equations can be obtained by imposing some extra condition on $v_{1}, \ldots, v_{M}$ :

$$
\sum_{j=1}^{N} \alpha_{j} v_{k}\left(\mathbf{x}_{j}\right)=0, \quad k=1, \ldots, M
$$

In many practical applications (in the case of MQ), it is observed that the term $\sum_{l=1}^{M} \gamma_{l} v_{l}(\mathbf{x})$ does not have a great effect on the accuracy of the method.

Our observations have shown that the errors are typically largest near the boundary compared to the errors in the domain far from the boundary. It therefore makes sense to impose more information there. Fedoseyev, Friedman and Kansa [18] formulated a method that collocates both with the boundary condition and the PDE at the boundary points.

In order to have a matching number of unknowns and equations, additional expansion functions are added. The centers of these are placed outside the boundary. Let the center points be denoted by $z_{j}$, where

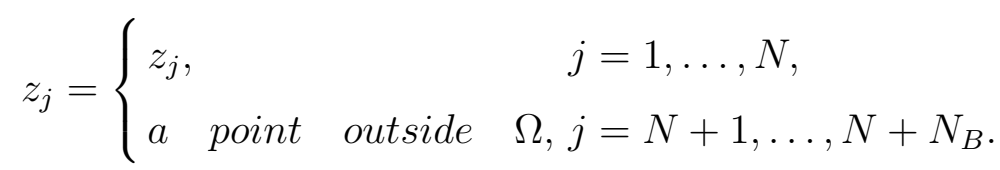

The RBF interpolant now takes the form

$$
u(\mathbf{x})=\sum_{j=1}^{N+N_{B}} \alpha_{j} \phi_{j}\left(\left\|\mathbf{x}-z_{j}\right\|\right) .
$$


The collocation equations are very similar to (3). The difference lies in the extra collocation at the boundary and the added centers. Practical application will be presented in the next chapter.

\section{MODELING OF THE RADIONUCLIDE MIGRATION}

Assessment of the release and the transport of long-lived radioactive nuclides from a repository to the biological environment is an important part of the safety analysis of repository concepts. In this assessment mathematical models describing the mechanisms involved in the nuclide transport from the repository to the biosphere are essential tools. For example, the groundwater models are mathematical representations of the flow of water and the transport of solutes in the subsurface. Models are used to compute the hydraulic head, velocity, concentration, etc., from hydrologic and mass inputs, hydrogeologic and mass-transfer parameters, and conditions at the boundary of the domain.

Groundwater models are presented by motion and continuity equations. The majority of the codes currently used or under development are based on the advective-dispersive equation with various physical phenomena added. According to this equation, mass transport is controlled by two mechanisms: advection and dispersion. Advection accounts for the movement of the solute, linked to the fluid by the water velocity. Water and solute velocity can be assessed through Darcy's law. Dispersion accounts for mixing caused by diffusion and by random departures from the mean stream.

The simulation area will be $2 \mathrm{D}$ rectangular with the Neumann and Dirichlet boundary conditions. The Neumann boundary conditions represent flow and no-flow while Dirichlet boundary conditions represent constant pressure and concentration.

\subsection{Laplace equation}

The first step of radionuclide transport modeling is to solve the Laplace equation to obtain the Darcy velocity. In this case the Neumann and Dirichlet boundary conditions will be defined along the boundary. Heterogeneous and anisotropic porous media and incompressible fluid are assumed. The equation has the following form [19]:

$$
K_{x} \frac{\partial^{2} p}{\partial x^{2}}+K_{y} \frac{\partial^{2} p}{\partial y^{2}}+\frac{\partial K_{x}}{\partial x} \frac{\partial p}{\partial x}+\frac{\partial K_{y}}{\partial y} \frac{\partial p}{\partial y}=0
$$

where $p[\mathrm{~Pa}]$ is the pressure of the fluid and $K_{x}\left[\frac{\mathrm{m}}{\mathrm{y}}\right]$ and $K_{y}\left[\frac{\mathrm{m}}{\mathrm{y}}\right]$ are the 
components of hydraulic conductivity tensor. The corresponding boundary conditions are:

$$
\frac{\partial p}{\partial x} s_{x}+\frac{\partial p}{\partial y} s_{y}=g_{1}(x, y)
$$

or

$$
p=g_{2}(x, y)
$$

where $s_{x}$ and $s_{y}$ are the components of the unit vector normal to the boundary. The Laplace equation was solved by using direct collocation [18]. We add an additional set of nodes (outside of the domain) adjacent to the boundary and add an additional set of collocation equations. The approximate solution is expressed as:

$$
p(x, y)=\sum_{j=1}^{N_{I}+2 N_{B}} \alpha_{j} \varphi_{j}(x, y)
$$

where $\alpha_{j}, j=1, \ldots, N_{I}+2 N_{B}$ are the unknown coefficients to be determined and $\varphi_{j}(x, y)=\sqrt{\left(x-x_{j}\right)^{2}+\left(y-y_{j}\right)^{2}+c^{2}}$ are Hardy's multiquadrics functions. By substituting (11) into (8), (9) or (10), we have:

$$
\begin{array}{r}
\left.\sum_{j=1}^{N_{I}+2 N_{B}}\left(K_{x} \frac{\partial^{2} \varphi_{j}}{\partial x^{2}}+K_{y} \frac{\partial^{2} \varphi_{j}}{\partial y^{2}}+\frac{\partial K_{x}}{\partial x} \frac{\partial \varphi_{j}}{\partial x}+\frac{\partial K_{y}}{\partial y} \frac{\partial \varphi_{j}}{\partial y}\right)\right|_{\left(x_{i}, y_{i}\right)} \alpha_{j}=0 \\
i=1,2, \ldots, N_{I}+N_{B}
\end{array}
$$

$$
\begin{aligned}
& \sum_{j=1}^{N_{I}+2 N_{B}}\left(\frac{\partial \varphi_{j}\left(x_{i}, y_{i}\right)}{\partial x} s_{x}+\frac{\partial \varphi_{j}\left(x_{i}, y_{i}\right)}{\partial y} s_{y}\right) \alpha_{j}=g_{1}\left(x_{i}, y_{i}\right) \\
& i=N_{I}+N_{B}+1, \ldots, N_{I}+2 N_{B}
\end{aligned}
$$

or

$$
\sum_{j=1}^{N_{I}+2 N_{b}} \varphi_{j}\left(x_{i}, y_{i}\right) \alpha_{j}=g_{2}\left(x_{i}, y_{i}\right), \quad i=N_{I}+N_{B}+1, \ldots, N_{I}+2 N_{B}
$$


The pressure gradient is evaluated by:

$$
\frac{\partial p}{\partial x}=\sum_{j=1}^{N_{I}+2 N_{B}} \alpha_{j} \frac{\partial \varphi_{j}(x, y)}{\partial x}, \quad \frac{\partial p}{\partial y}=\sum_{j=1}^{N_{I}+2 N_{B}} \alpha_{j} \frac{\partial \varphi_{j}(x, y)}{\partial y}
$$

For the calculation of velocity in principal directions we use Darcy's law [19]:

$$
v_{x}=-\frac{K_{x}}{\omega \rho a} \frac{\partial p}{\partial x}, \quad v_{y}=-\frac{K_{y}}{\omega \rho a} \frac{\partial p}{\partial y}
$$

where $\rho$ is the density of the fluid, $\omega$ is porosity and $a$ is gravitational acceleration.

\subsection{Advection-Dispersion Equation}

In the next step, the velocities obtained from the Laplace equation are used in the advection-dispersion equation. The advection-dispersion equation for transport through the heterogeneous saturated porous media zone at a macroscopic level with retardation and decay is [19]:

$$
\begin{array}{cc}
R \frac{\partial u}{\partial t}=\left(\frac{D_{x}}{\omega} \frac{\partial^{2} u}{\partial x^{2}}+\frac{D_{y}}{\omega} \frac{\partial^{2} u}{\partial y^{2}}+\frac{\partial D_{x}}{\partial x} \frac{\partial u}{\partial x}+\frac{\partial D_{y}}{\partial y} \frac{\partial u}{\partial y}\right)- \\
-v_{x} \frac{\partial u}{\partial x}-v_{y} \frac{\partial u}{\partial y}-R \lambda u, \quad(x, y) \in \Omega, & 0 \leq t \leq T, \\
\left.u\right|_{(x, y) \in \partial \Omega}=g(x, y, t), & 0 \leq t \leq T \\
\left.u\right|_{t=0}=h(x, y), & (x, y) \in \Omega,
\end{array}
$$

where $x$ is the Eulerian groundwater flow axis and $y$ is the Eulerian transverse axis in the $2 \mathrm{D}$ problem, $u$ is the concentration of contaminant in the groundwater $\left[\mathrm{Bqm}^{-3}\right], D_{x}$ and $D_{y}$ are the components of the dispersion tensor $\left[\mathrm{m}^{2} \mathrm{y}^{-1}\right]$ in the saturated zone, $\omega$ is porosity of the saturated zone $[-], v_{x}$ and $v_{y}$ are components of velocity vector $\left[\mathrm{my}^{-1}\right]$ at interior points, $R$ is the retardation factor in the saturated zone $[-]$ and $\lambda$ is the radioactive decay constant $\left[\mathrm{y}^{-1}\right]$. In these cases $[y]$ means years. For the parabolic problem, we consider the implicit scheme: 


$$
\begin{array}{r}
R \frac{u^{n+1}-u^{n}}{\delta t}=\left(\frac{D_{x}}{\omega} \frac{\partial^{2} u^{n+1}}{\partial x^{2}}+\frac{D_{y}}{\omega} \frac{\partial^{2} u^{n+1}}{\partial y^{2}}\right. \\
\left.+\frac{\partial D_{x}}{\partial x} \frac{\partial u^{n+1}}{\partial x}+\frac{\partial D_{y}}{\partial y} \frac{\partial u^{n+1}}{\partial y}\right)- \\
-v_{x} \frac{\partial u^{n+1}}{\partial x}-v_{y} \frac{\partial u^{n+1}}{\partial y}-R \lambda u^{n+1}
\end{array}
$$

where $\delta t$ is the time step and $u^{n}$ and $u^{n+1}$ are the contaminant concentrations at the time $t_{n}$ and $t_{n+1}$. The approximate solution is expressed as:

$$
u\left(x, y, t_{n+1}\right)=\sum_{j=1}^{N} \alpha_{j}^{n+1} \varphi_{j}(x, y)
$$

where $\alpha_{j}^{n+1}, j=1, \ldots, N$ are the unknown coefficients to be determined and $\varphi_{j}(x, y)=\sqrt{\left(x-x_{j}\right)^{2}+\left(y-y_{j}\right)^{2}+c^{2}}$ are Hardy's multiquadrics functions.

By substituting (19) into (17), we obtain:

$$
\begin{array}{r}
\left.\sum_{j=1}^{N}\left(R \frac{\varphi_{j}}{\delta t}-\frac{D_{x}}{\omega} \frac{\partial^{2} \varphi_{j}}{\partial x^{2}}-\frac{D_{y}}{\omega} \frac{\partial^{2} \varphi_{j}}{\partial y^{2}}-\frac{\partial D_{x}}{\partial x} \frac{\partial \varphi_{j}}{\partial x}-\frac{\partial D_{y}}{\partial y} \frac{\partial \varphi_{j}}{\partial y}\right)\right|_{\left(x_{i}, y_{i}\right)} \alpha_{j}^{n+1}+ \\
+\left.\sum_{j=1}^{N}\left(v_{x} \frac{\partial \varphi_{j}}{\partial x}+v_{y} \frac{\partial \varphi_{j}}{\partial y}+R \lambda \varphi_{j}\right)\right|_{\left(x_{i}, y_{i}\right)} \alpha_{j}^{n+1}=R \frac{u^{n}\left(x_{i}, y_{i}\right)}{\delta t}, \quad i=1,2, \ldots, N_{I}
\end{array}
$$

$$
\sum_{j=1}^{N} \varphi_{j}\left(x_{i}, y_{i}\right) \alpha_{j}^{n+1}=g\left(x_{i}, y_{i}, t_{n+1}\right), \quad i=N_{I}+1, \ldots, N
$$

The system of linear equations $((20)-(21))$ for the unknown $\alpha_{j}^{n+1}, j=1, \ldots, N$ has to be solved, where $N=N_{I}+N_{B}$ is the number of collocation points, $N_{I}$ is the number of interior points and $N_{B}$ is the number of boundary points. Equation (19) gives the approximate solution at any point in the domain $\Omega$.

\section{NUMERICAL EXAMPLE}

The simulation was implemented for a rectangular area which was $600 \mathrm{~m}$ long and $300 \mathrm{~m}$ wide. The source (initial condition) was Thorium $(T h-230)$ with activity $1 \cdot 10^{6} \mathrm{~Bq}$ and half life of 77,000 years. The location of the radioactive source is presented in Fig. 1. (symbol $\bullet$ in Fig. 1). 


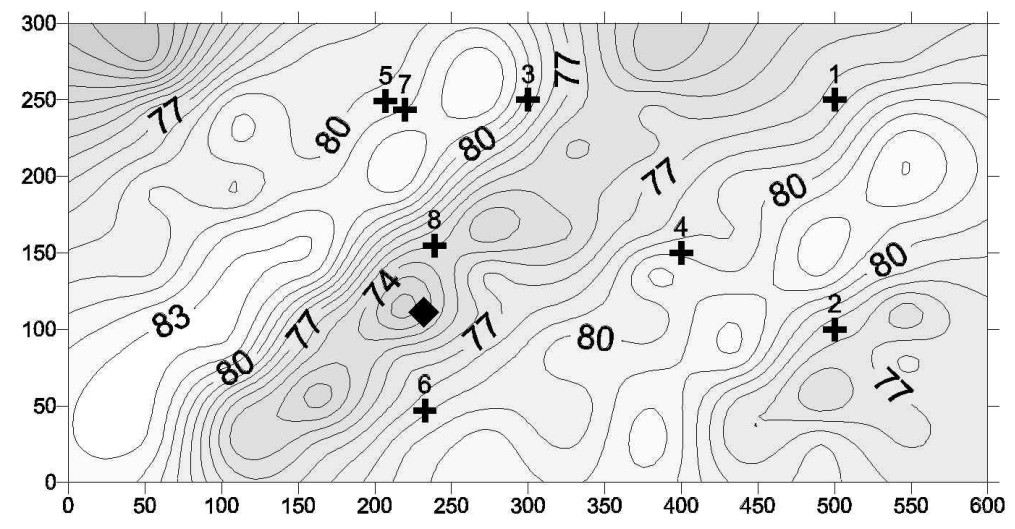

Fig. 1: Distribution of hydraulic conductivity based on 8-point data set

The groundwater flow field is presented for steady-state conditions. Except for the inflow (left side) and outflow (right side), all boundaries have a noflow condition $\frac{\partial p}{\partial s}=0$ ( $s$ is normal to the boundary). The inflow rate was 1 $\mathrm{m} / \mathrm{y}$. At the outflow side, time-constant pressures at the boundaries were set. Longitudinal dispersivity $a_{x}$ is $200 \mathrm{~m}$ and transversal dispersivity $a_{y}$ is $20 \mathrm{~m}$. For the porosity $\omega$ we used values 0.25 . The retardation constant $R$ is 800 .

The traditional finite difference scheme was also used for solving the Laplace and advection-dispersion equation. For the approximation of the first derivative second-order central difference or one-sided difference were used. But for the approximation of the second derivatives we used the second-order central second difference [20], [21]. The time dependent part we implemented with the implicit scheme. To implement a finite difference method we constructed a grid where the horizontal dimension represents discrete points of the variable $x$ with constant step $\Delta x$ while vertical dimension represents discrete points with constant step $\Delta y$. The discretization grid has actually $12 \times 12$ points. Thus we had: $N=144, N_{I}=100$ and $N_{B}=44$.

One of the difficulties encountered in applying these models to make predictions involves securing good estimates of the hydrogeologic parameters. In practice, our objective is to estimate a field variable $z(\mathbf{x})$ over a region. Usually, because of scarcity of information, we cannot find a unique solution. It is useful to think of the actual unknown $z(\mathbf{x})$ as one of a collection of possibilities $z(\mathbf{x} ; 1), z(\mathbf{x} ; 2), \ldots$ This collection (ensemble) defines all possible solutions to our estimation problem. The ensemble of realizations with their probabilities defines what is known as the spatial stochastic process. We used the averaging process since specifying all possible solutions and their probabilities is not an easy task, and it is more convenient to specify and to work with ensemble averages or statistical moments (mean and covariance function). The quality of the results also depends on the quality of input data. The hydraulic conductivity was modelled as a random field with mean and covariance functions. 
In mathematical terms, the most convenient method for simulation is sequential Gaussian simulation because all successive conditional distributions from which simulated values are drawn are Gaussian with parameters determined by the solution of a simple kriging system.

Sequential Gaussian simulation procedure [22]:

(1) First, use a sequential Gaussian simulation to transform the data into a normal distribution.

(2) Then perform variogram modelling on the data. Select one grid node at random, then krige the value at that location. This will also give us the kriged variance.

(3) Then draw a random number from a normal distribution that has a variance equivalent to the kriged variance and a mean equivalent to the kriged value. This number will be the simulated number for that grid node.

(4) Select another grid node at random and repeat. For the kriging, include all the previously simulated nodes to preserve the spatial variability as modelled in the variogram.

(5) When all nodes have been simulated, back transform to the original distribution. This gives us first realization using a different random number sequence to generate multiple realizations of the map.

Following the above procedure, hydraulic conductivity was generated at different points based on two different sets of input data. In the first one, hydraulic conductivity at 8 different points is given (values are: 66.00, 71.00, 73.00, 75.00, $\left.76.52,77.02,79.74,83.41\left[\frac{\mathrm{m}}{\mathrm{y}}\right]\right)$. The distribution of hydraulic conductivity for one specific simulation is shown in Fig. 1. The coordinates of these values are also presented in Fig. 1 and marked with "+". In Fig. 1 we cannot see much variability of hydraulic conductivity. One of the reasons could be that there are not many differences between the prescribed values of hydraulic conductivity. The following variogram parameters are chosen: positive variance contribution or sill is equal to 1.0 and nugget effect is 0.0 . Simple kriging is chosen as the type of kriging. A spherical model is chosen as a type of variogram structure. The element defining the geometric anisotropy is range. The maximum horizontal range is $600 \mathrm{~m}$ and the minimum horizontal range is $300 \mathrm{~m}$. It is assumed that the mean in the case of simple kriging is known.

In the second case the database of 16 different points is used (values are: 66.00, $71.00,73.00,75.00,76.52,77.02,79.74,83.41,86.00,90.00,93.00,95.00,96.52$, $\left.100.02,107.74,110.00\left[\frac{m}{y}\right]\right)$. The coordinates of these values are also presented in Fig. 2 and marked with "+". It is also necessary to stress out that the first 8 points of the 16 point set are those of the 8 point set. Positive variance contribution or sill size 0.7 and nugget effect size 0.3 as variogram parameters are chosen. Ordinary kriging is chosen as the type of kriging where the constant mean value is replaced by the location-dependent estimate. 


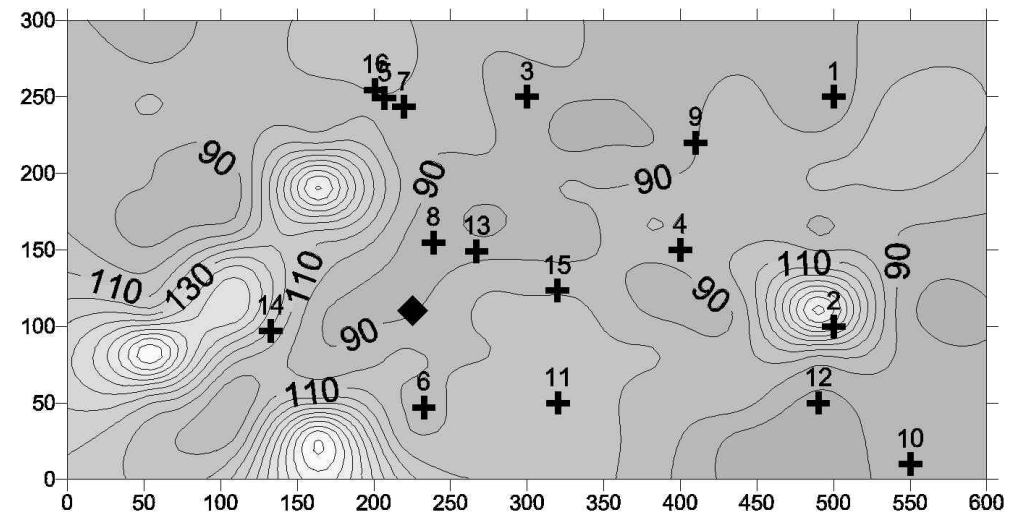

Fig. 2: Distribution of hydraulic conductivity based on 16-point data set

Distribution of average of contaminant concentrations (8 points) for radial basis function method and distribution of average of contaminant concentrations (8 points) for FDM are shown in Figs. 3 and 4. Distribution of standard deviation of contaminant concentrations ( 8 points) for radial basis function method and distribution of standard deviation of contaminant concentrations (8 points) for FDM are shown in Figs. 5 and 6. We can see that concentration clouds in Fig. 4 are wider in the y direction than concentration clouds in Fig. 3.

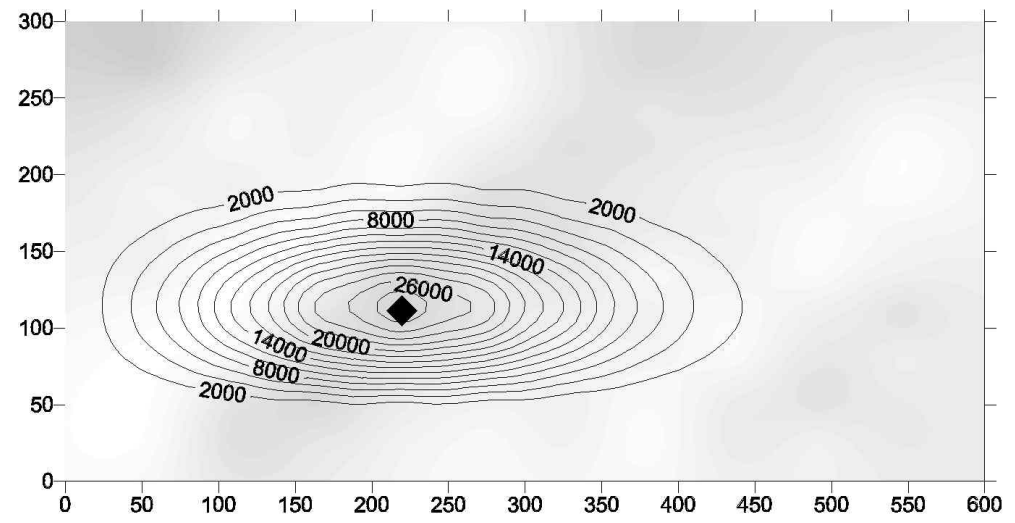

Fig. 3: Distribution of average of contaminant concentrations (8 points) (Radial basis function) 


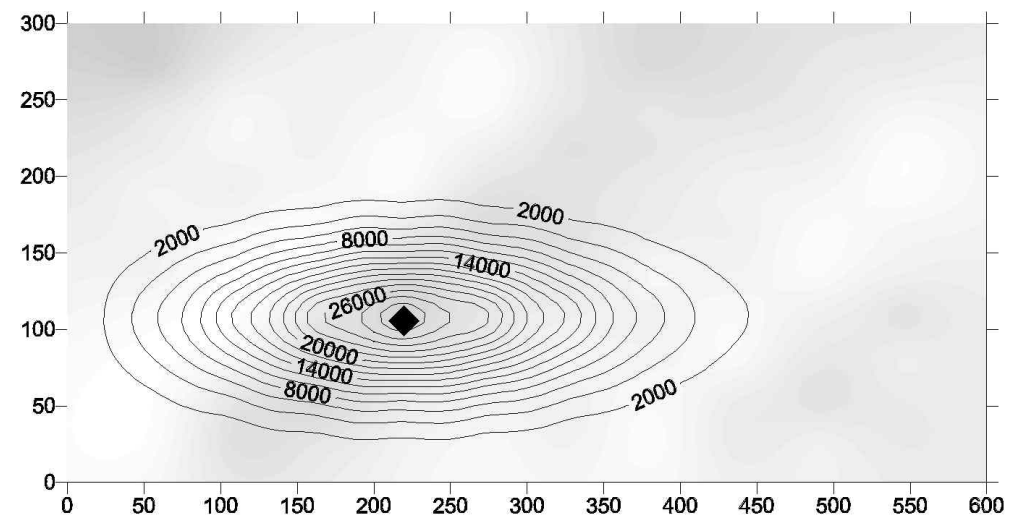

Fig. 4: Distribution of average of contaminant concentrations (8 points) (Finite difference method)

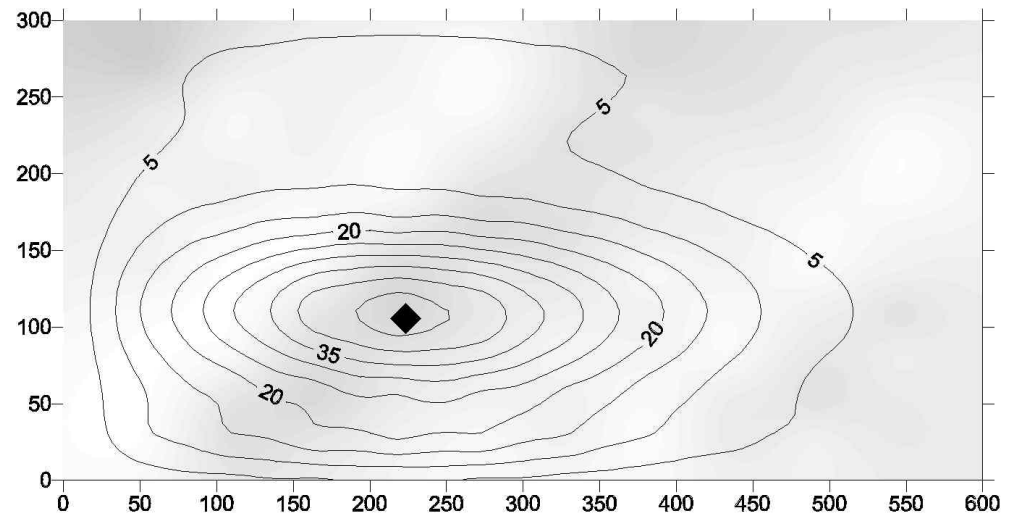

Fig. 5: Distribution of standard deviation of contaminant concentrations (8 points) (Radial basis function)

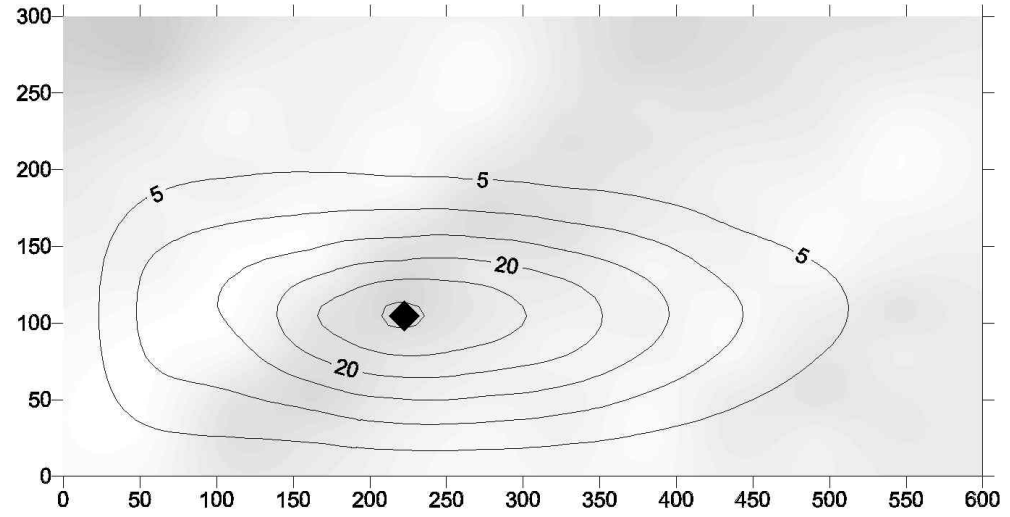

Fig. 6: Distribution of standard deviation of contaminant concentrations (8 points) (Finite difference method)

Possible reason for this is that the central difference schemes do not always follow the proper flow of information throughout the flow field. In many cases, 
they draw numerical information from outside the domain of dependence of a given grid point [21]. That mean that properties at grid point e. g. $i$ should depend only on the upstream flow field, i.e., on properties at grid poin $i-1$. Grid point $i-1$ is within the domain of dependence of point $i$. The properties at grid point $i+1$ do not physically influence point $i$, and a proper numerical scheme should reflect this fact.

Distribution of average of contaminant concentrations (16 points) for radial basis function method and distribution of average of contaminant concentrations (16 points) for FDM after 100, 000 years are shown in Figs. 7 and 8.

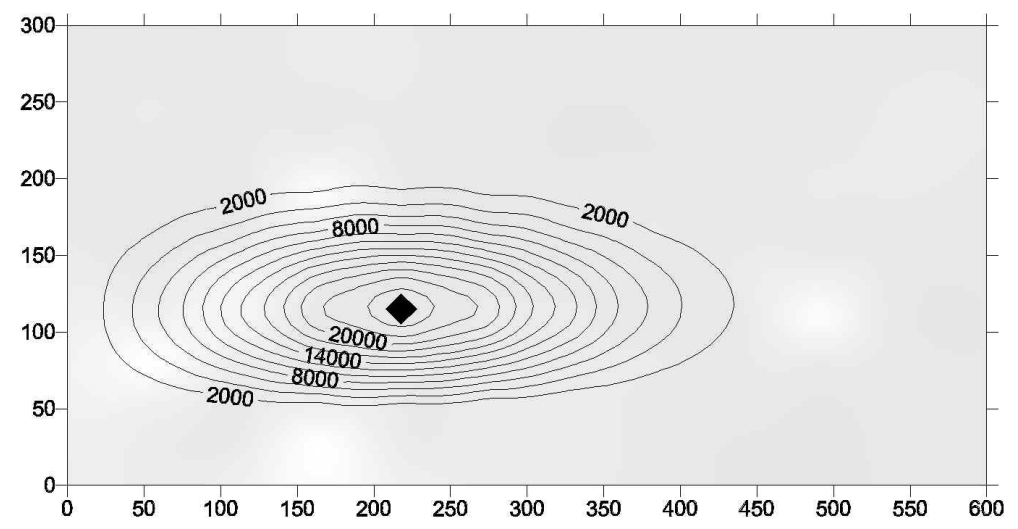

Fig. 7: Distribution of average of contaminant concentrations (16 points) (Radial basis function)

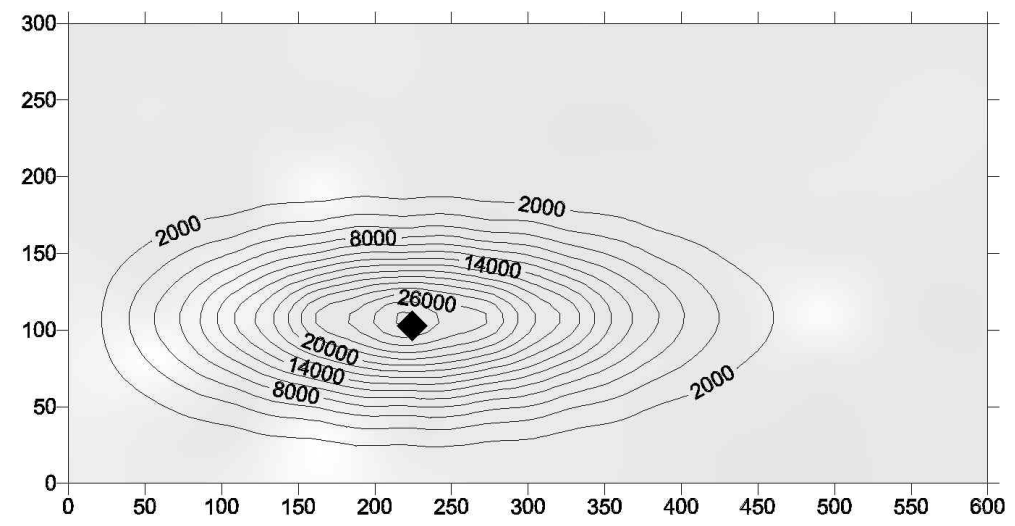

Fig. 8: Distribution of average of contaminant concentrations (16 points) (Finite difference method)

Distribution of standard deviation of contaminant concentrations (16 points) for radial basis function method and distribution of standard deviation of contaminant concentrations (16 points) for FDM are shown in Figs. 9 and 10. 


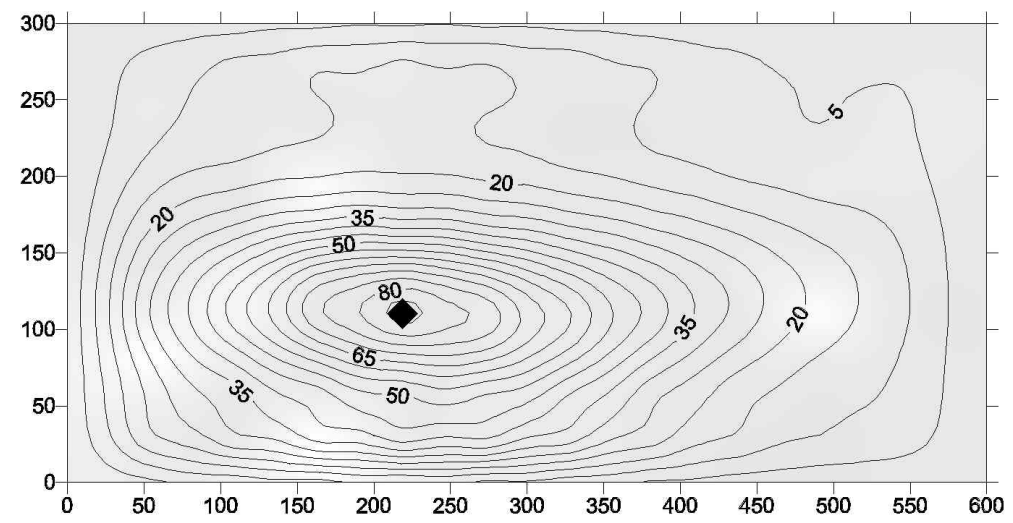

Fig. 9: Distribution of standard deviation of contaminant concentrations (16 points) (Radial basis function)

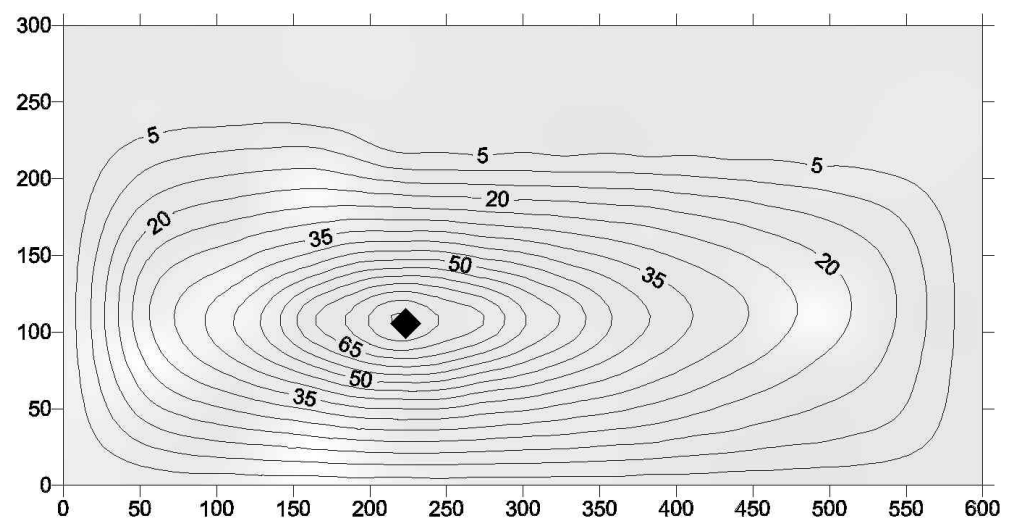

Fig. 10: Distribution of standard deviation of contaminant concentrations (16 points) (Finite difference method)

The same situation is evident here. We can also see that concentration clouds in Fig. 8 are wider in the y direction than concentration clouds in Fig. 7. The reason for this is that FDM includes upwinding. In other words, a finite difference introduces artificial numerical dispersion that can be very significant as compared to the physical dispersion.

FDM and the radial basis function method are both applied to the calculation of concentrations, average and standard deviation through 100 simulations. For the purpose of comparing FDM and the Kansa method, we plotted differences (Fig. 11 and Fig. 12). The so-called normalized error was defined symbolically as:

$$
\frac{\left|u_{F D M}-u_{R B F}\right|}{\max \left(u_{F D M}, u_{R B F}\right)}
$$

where $u_{F D M}$ is the value calculated with FDM and $u_{R B F}$ is the value calculated 
with RBF.

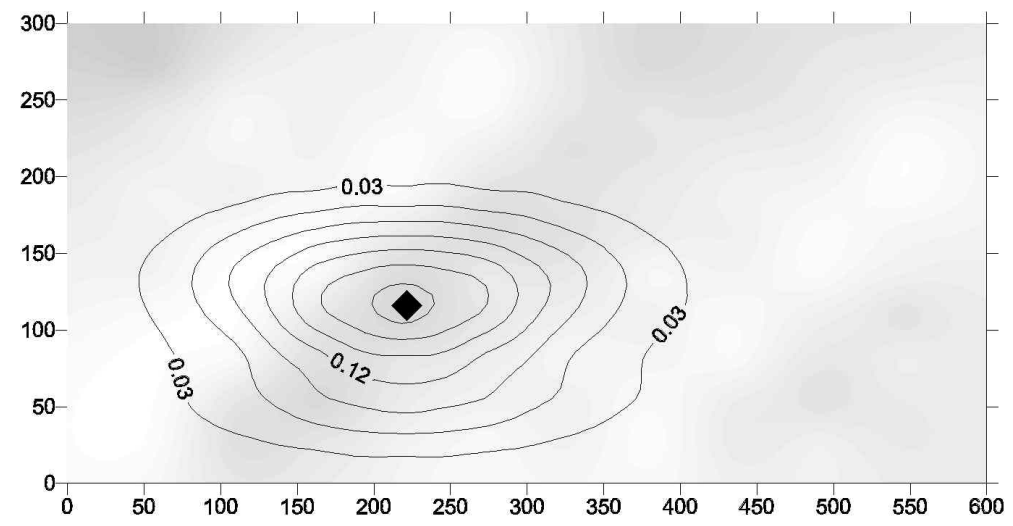

Fig. 11: Normalized error (8 points)

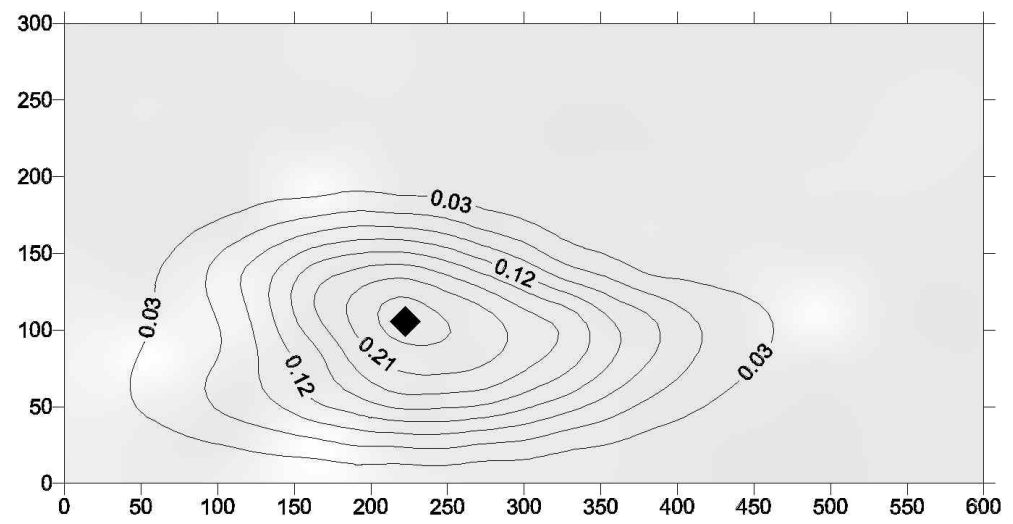

Fig. 12: Normalized error (16 points)

In the case of hydraulic conductivity based on an 8-point data set and a 16point data set, the normalized error is generally low (below $6 \%$ ) with exception in the region with higher concentration (Fig. 11 and Fig. 12). Comparison of normalized errors shows that the maximal value of normalized error in Fig. 12 is higher than the maximal value of normalized error in Fig. 11. We noticed that the standard deviation is also relatively large in the region with higher concentration (Fig. 9 and 10). The reason for differences we can find in influence of geostatistical parameters to choice of a shape parameter.

In our problem we used multiquadric (MQ). MQ's performance depends on the choice of shape parameter $c$. In the past, there have been several numerical experiments and empirical formulas that suggest how to chose value of such parameters, which in general depend on the density of the interpolation centres. Hardy [23] showed a good recipe for a safe choice of this shape parameter to be about $85 \%$ of the average distance between pairs of points. Carlson and Foley [24] showed that as the shape parameter increases, the errors decrease until ill-conditioning renders the solutions unstable. Kansa and Hon 
[25] showed domain decomposition controls the ill-conditioning problem.

In practice, the optimal value of the shape parameter can be determined by numerical experiments. The optimal shape parameter depends on the properties of numerical solution, number and locations of the collocations points. Therefore, a question how to find the optimal shape parameter for arbitrary real problem given by geometry and hydrological parameters of continuum always appears as one of the key problems. The shape parameter was chosen according to the graphic procedure [26] where we tried to answer the question how to find good optimal shape parameter, which fulfils the equation in more points. Many realizations of the equations were made using different shape parameters at different points and different geostatistical parameters. We calculated residual errors from the equation and boundary conditions at different shape parameters, and tried to find minimum residual errors whose shape parameter was chosen as optimal.

\section{CONCLUSION}

This work presents modelling of radionuclide migration through the geosphere using the radial basis function method, finite difference method and geostatistics. The influence of geostatistical data on the reliability and accuracy of computational modelling of a mass transport problem was also investigated. The only physical data modelled as a random field was hydraulic conductivity of the investigated field.

In the case of radionuclide migration, two evaluation steps were performed. In the first step the velocities in principal directions were determined from the pressure of the fluid obtained from the Laplace differential equation. In the second step the advection-dispersion equation was solved to find the concentration of the contaminant. In this case the method of evaluation was verified by comparing results with those obtained from the finite difference method.

Results show that differences exist between both numerical schemes. Different sets of input data yield differences between schemes. In the simulation, a very large scatter caused by different given values of hydraulic conductivity was observed. Another reason for differences could be the result of which kriging method was used to apply in the sequential Gaussian methods.

We conclude that the Kansa method is a valid alternative to the FDM because of its simpler implementation. The only geometric properties that are used in an RBF approximation are the pair-wise distances between points. Distances are easy to compute in any number of space dimensions, so working in higher dimensions does not increase the difficulty. The presence of a shape parameter offers the opportunity to exploit its value to obtain a better solution. Results show that the RBF solution has far less diffusion than the finite difference method that includes upwinding. For public safety, we do not want numer- 
ical dispersion under-predicting the concentration contours; nor do we want to show that the concentrations of radionuclides are more dilute than they actually are.

Acknowledgements - The authors would like to thank the Slovenian Nuclear Safety Administration for their support and understanding. 


\section{References}

1. L. B. Lucy, A numerical approach to the testing of the fission hypothesis, Astro. J. 8, 1013-1024, (1977).

2. B. Nayroles, G. Touzot, P. Villon, Generalizing the finite element method: diffuse approximation and diffuse elements, Comput. Mech., 10, 307-318, (1992).

3. T. Belytschko, Y.Y Lu, L. Gu, Element-free Gelerkin methods, Int. J. Numer. Meth. Engrg., 37, 229-256, (1994).

4. W. Liu, S. Jun, Y. Zhang, Reproducing kernel particle methods, Int. J. Numer. Meth. Fluids. 20, 1081-1106, (1995).

5. I. Babuska, J. Melenk, The partition of unity method, Int. J. Numer. Meth. Engrg, 40, 727-758, (1997).

6. C. A. Duarte, J. T. Oden, Hp clouds-a meshless method to solve boundary-value problems, TICAM Report 95-05, (1995).

7. E. Onate, S. Idelsohn, O. C. Zienkiewicz, R. L. Taylor, A finite point method in computational mechanics, Application to convective transport and fluid flow, Int. J. Numer. Meth. Engrg., 39, 3839-3866, (1996).

8. S. N. Atluri, T. Zhu, New meshless local Petrov-Galerkin (MLPG) approach in computational mechanics, Comput. Mech., 22(2), 117-127, (1998).

9. D. Nardini, D., C. A. Brebbia, A new approach to free vibration analysis using boundary elements, in Boundary element Methods in Engineering, C. A. Brebbia (Ed.), Springer-Verlag, Berlin, (1982).

10. G. Fairweather, A. Karageorghis, The method of fundamental solutions for elliptic boundary value problems, Adv. Comput. Math., 9(1/2), 69-95, (1998).

11. M. A. Golberg, C. S. Chen, The method of fundamental solutions for potential, Helmholtz and diffusion problems, in Boundary Integral Methods-Numerical and Mathematical Aspects, M. A. Golberg (Ed.), Computational Mechanics Publications, p. 103-176, (1998).

12. E. J. Kansa, Multiquadrics-A scattered data approximation scheme with applications to computational fluid dynamics-I. Surface approximations and partial derivative estimates, Computers Math. Applic. 19 (8/9), 127-145, (1990).

13. E. J. Kansa, Multiquadrics-A Scattered data approximation scheme with applications to computational fluid dynamics-II. Solutions to parabolic, hyperbolic and elliptic partial differential equations, Computers Math. Applic. 19 (8/9), 147-161, (1990).

14. Y. C. Hon, M. W. Lu, W. M. Xue, Y. M. Zhu, Multiquadric method for the numerical solution of a biphasic mixture model, Appl. Math. Comput., 88, 153175, (1997). 
15. G. E. Fasshauer, Solving partial differential equations by collocation with radial basis functions, Proceeding of Chamonix, Vanderbilt University Press, Nashville, (1996).

16. R. L. Hardy, Multiquadric equation of topography and other irregular surfaces, J. Geophys. Res., 176, 1905-1915, (1971).

17. M. D. Buhmann, Radial basis functions, Cambridge University Press (2003).

18. A. I. Fedoseyev, M. J. Friedman and E. J. Kansa, Improved multiquadric method for elliptic partial differential equations via PDE collocation on the boundary, Computers Math. Applic. 43, 439-455, (2002).

19. J. Bear and A. Verruijt, Modeling Groundwater Flow and Pollution, D. Reidel Publishing Company, Dordrecht, Holland, (1987).

20. G. D. Smith, Numerical Solution of Partial Differential Equations: Finite Difference Methods, Oxford Applied Mathematics and Computing Science Series, Oxford University Press, (1978).

21. J. D. Anderson, Jr., Computational Fluid Dynamics: The Basis with Applications, McGraw-Hill, Inc., New York, (1995).

22. C. V. Deutsch, A. G. Journel, GSLIB Geostatistical Software Library and User's Guide, Oxford University Press, (1998).

23. R. L. Hardy, Theory and Applications of The Multiquadric-Biharmonic Method. 20 Years of Discovery 1968-1988, Computer and Mathematics with Applications, 10 (8-9), 163-208, (1990).

24. R. E. Carlson and T. A. Foley, The Parametr R2 in Multiquadric and Related Interpolations, Computers Math. Applic., 21, 29-42, (1991).

25. E. J. Kansa and Y. C. Hon, Circumventing The Ill-Conditioning Problem with Multiquadric Radial Basis Functions: Applications to Elliprtic Partial Differential Equations, Computers Math. Applic., 39 (7/8), 123-137, (2000).

26. L. Vrankar, G. Turk and F. Runovc, Modelling of Radionuclide Migration through the Geosphere with Radial Basis Function Method and Geostatistics, Journal of the Chinese Institute of Engineers, Vol. 27, No. 4, pp. 455-462, (2004). 